\title{
Enteroscopia de doble balón: experiencia inicial en un hospital universitario
}

\author{
Double balloon enteroscopy: Initial experience in a university hospital
}

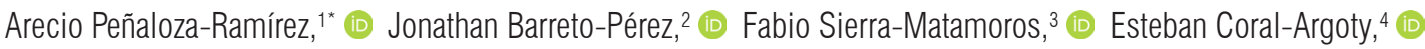 \\ Alejandra Castro-Rodríguez, ${ }^{5}$ (D) Pedro Aponte-Ordónez. ${ }^{6}$ (b)
}

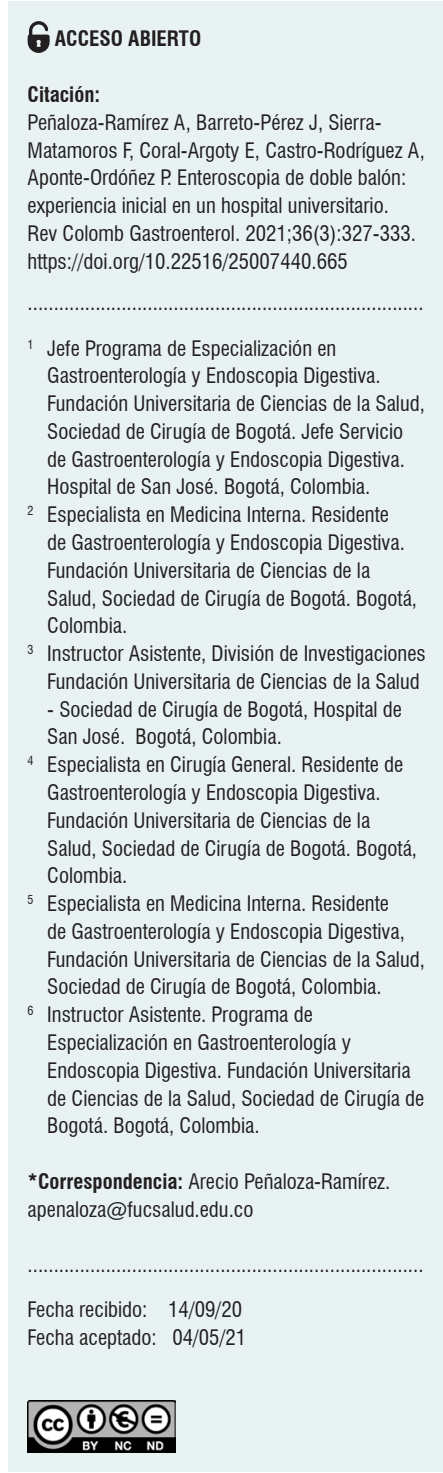

\begin{abstract}
Resumen
Introducción: el estudio de intestino delgado es un desafío, motivo de avance médico y de interés. La enteroscopia de doble balón (EDB) permite una visualización directa del intestino delgado. Objetivo: describir indicaciones, características del procedimiento, hallazgos, complicaciones y seguimiento de pacientes llevados a EDB en el Hospital de San José de Bogotá, entre noviembre de 2011 y abril de 2019. Metodología: estudio de serie de casos. Se incluyeron pacientes con indicación clínica o por imágenes diagnósticas sugerentes de lesión a nivel de intestino delgado. Se empleó estadística descriptiva. Resultados: se realizaron 45 enteroscopias a 44 pacientes, con una mediana de edad de 58 años. La principal indicación fue hemorragia digestiva potencial de intestino delgado $(53,3 \%)$ y diarrea crónica $(11,1 \%)$; fueron más frecuentes los procedimientos por vía anterógrada. La mayoría de los resultados fue normal (46,7 \%); entre los hallazgos más frecuentes estuvieron los tumores (11,1\%), enfermedad de Crohn (8,9\%) y úlceras/erosiones $(6,7 \%)$. El rendimiento diagnóstico fue 53,3\%, la correlación con la videocápsula endoscópica (VCE) fue 37,5 \% y con estudios de imagen, $42,9 \%$. No se presentaron complicaciones. Se hizo seguimiento al $91,1 \%$ de los pacientes con una mediana de 56 meses y se observó la resolución de síntomas en el 78,1 \%. La finalidad de la enteroscopia fue diagnóstica en el 84,4\%. Conclusiones: La EDB es útil en la evaluación del intestino delgado con posibilidad terapéutica. Los resultados son similares a los reportados en la literatura mundial. Si se realiza seguimiento, se puede definir la necesidad de repetición del procedimiento, realización de estudios adicionales u observar resolución de los síntomas.
\end{abstract}

\section{Palabras clave}

Enteroscopia de doble balón, hemorragia gastrointestinal, videocápsula endoscópica.

\section{Abstract}

Introduction: The study of the small bowel is a challenging task that has sparked interest and progress in medicine. In this context, double balloon enteroscopy (DBE) has allowed the direct visualization of the small intestine. Objective: To describe indications, procedure characteristics, findings, complications, and followup of patients taken to DBE at the Hospital de San José, Bogotá, between November 2011 and April 2019. Methodology: Case series study. Patients with a clinical indication or diagnostic images suggestive of small bowel lesion were included. Descriptive statistics were used. Results: 45 enteroscopies were performed in 44 patients, with a median age of 58 years. The main indication was potential small bowel bleeding $(53.3 \%)$ and chronic diarrhea (11.1\%). Anterograde procedures were more frequent. Most of the results were normal (46.7\%); tumors (11.1\%), Crohn's disease (8.9\%), and ulcers/erosions (6.7\%) were among the most frequent findings. The diagnostic yield was $53.3 \%$ and the correlation with capsule endoscopy (CE) was $37.5 \%$, and with imaging studies $42.9 \%$. There were no complications. $91.1 \%$ of patients were followed up on after a median of 56 months, with $78.1 \%$ reporting symptom remission. The aim of enteroscopy was diagnostic in $84.4 \%$. Conclusions: DBE is useful in the evaluation of the small intestine with a therapeutic possibility. The results are similar to those reported in the world literature. Follow-up can define the need for repeating the procedure, performing further studies, or symptom resolution.

\footnotetext{
Keywords

Double Balloon Enteroscopy; Gastrointestinal Bleeding; Capsule Endoscopy.
} 


\section{INTRODUCCIÓN}

Durante mucho tiempo el intestino delgado se consideró "la caja negra" para los gastroenterólogos dada la imposibilidad de su visualización endoscópica por un método no quirúrgico; esto se debía a su longitud, posición anatómica y tamaño ${ }^{(1)}$. En el siglo XXI se desarrollaron técnicas endoscópicas que permitieron estudiar el intestino delgado sin necesidad de cirugía ${ }^{(2,3)}$. Entre los métodos de estudio se encuentran la videocápsula endoscópica (VCE), la enteroscopia con sus diferentes técnicas y los estudios de imagen.

La VCE se considera un estudio de primera línea con un alto valor predictivo negativo, pero con la limitación de no poder realizar intervenciones terapéuticas ${ }^{(4)}$. Una técnica complementaria es la enteroscopia, la cual debe realizarse si los hallazgos de la VCE u otros estudios de imagen son positivos, ya que cuenta con posibilidad terapéutica (biopsia, coagulación, polipectomía, entre otras $)^{(5)}$. La enteroscopia es invasiva, requiere sedación y es difícil en pacientes con adherencias ${ }^{(6)}$. Algunos estudios muestran que la VCE y la enteroscopia de doble balón (EDB) tienen rendimientos diagnósticos comparables hasta de un $60 \%{ }^{(4)}$.

Entre los métodos que se han desarrollado se encuentran la EDB introducida en 2001, de un solo balón en 2007, en espiral en 2008 y enteroscopia asistida por balón de alcance

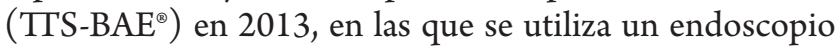
estándar sin necesidad de sobretubo. En general, estas técnicas tienen rendimientos diagnósticos comparables ${ }^{(7,8)}$ y su elección depende de la experiencia y disponibilidad local. No se han reportado diferencias en el rendimiento diagnóstico, terapéutico o tasa de complicaciones entre la enteroscopia en espiral y $\operatorname{EDB}^{(9,10)}$ y entre la enteroscopia de un solo balón y $\operatorname{EDB}^{(11-13)}$.

Existen dos posibilidades de ingreso. La vía se escoge dependiendo de la presentación clínica y revisión de estudios previos. Hasta en el $85 \%$ de los casos se logra un examen completo del intestino delgado cuando se utilizan las dos vías ${ }^{(14,15)}$. Sus complicaciones son bajas y están más relacionadas con la realización de procedimientos terapéuticos entre las que están la pancreatitis $(2 \%)$ y la perforación $(1 \%)^{(4)}$. La enteroscopia intraoperatoria es un procedimiento diagnóstico y eventualmente terapéutico útil pero invasivo y en general su uso debe limitarse a escenarios con dificultad para realizar una enteroscopia; bien sea por disponibilidad, cirugía previa o adherencias severas ${ }^{(14)}$.

La principal indicación es el sangrado potencial del intestino delgado, que representa el $5 \%$ de las causas de hemorragia digestiva ${ }^{(4)}$; se denomina de esta manera porque con el uso de todas las herramientas diagnósticas es posible establecer la causa del sangrado en un $75 \%$ de las veces, mientras que se diagnostica sangrado intestinal oscuro en el paciente con estudios endoscópicos y de intestino delgado negativos $^{(16-18)}$.

El estudio del sangrado del intestino delgado es difícil, puede ser recurrente y se relaciona con neoplasias, especialmente en pacientes menores de 40 años quienes tienen más probabilidades de tumores de intestino delgado (linfoma, tumores carcinoides, adenocarcinoma y poliposis hereditaria $)^{(14)}$. Es necesario un algoritmo diagnóstico que utilice de manera racional las herramientas disponibles localmente. Teniendo en cuenta lo anterior, es indispensable realizar estudios en pacientes llevados a EDB que permitan conocer las indicaciones, hallazgos, complicaciones y relación con otros métodos diagnósticos, así como su impacto en los desenlaces.

\section{MÉTODOS}

Estudio observacional descriptivo tipo serie de casos. La recolección de la información se realizó de manera prospectiva. Se incluyeron pacientes mayores de 18 años, con indicación clínica o por imágenes diagnósticas que sugerían lesión a nivel de intestino delgado, a quienes se les realizó EDB. Se excluyeron las enteroscopias intraoperatorias. La EDB se realizó con un enteroscopio Fujinon EN-450T5 $5^{\circ}$ según la técnica usual y sin el uso de fluoroscopia (la cual en la mayoría de estudios no se utiliza).

Se recolectó información correspondiente a variables sociodemográficas (sexo y edad) y del procedimiento que incluyeron indicación, duración, vía de acceso definida de acuerdo con la clínica del paciente, estudios de imagen o VCE, distancia explorada (se calculó por vía anterógrada en centímetros a partir del píloro y por vía retrógrada en centímetros a partir de la válvula ileocecal, sumando los avances y restando los retrocesos de manera aproximada), hallazgos, resultado de las biopsias, correlación con otros estudios y estado clínico del paciente en el seguimiento de rutina. Las variables cualitativas se describieron por medio de frecuencias absolutas y relativas. Las variables cuantitativas se describieron por medio de medidas de tendencia central y dispersión: medianas y RIC si la distribución no fue normal, o medias y desviaciones estándar (DE) en caso contrario. Se consideró rendimiento diagnóstico como la frecuencia relativa de hallazgos anormales y la correlación de hallazgos similares entre la EDB con la VCE o estudios de imagen.

\section{CONSIDERACIONES ÉTICAS}

El protocolo fue aprobado por el comité de ética del Hospital de San José de Bogotá y el comité de investigaciones de la Fundación Universitaria de Ciencias de la Salud. 
Este trabajo no implica la realización adicional de procedimientos, pero sí utiliza la información procedente de ellos. Tampoco se espera algún beneficio adicional dado que su alcance es descriptivo. Además, se clasifica como una investigación sin riesgo según la resolución 8430 de 1993 del Ministerio de Salud colombiano.

\section{RESULTADOS}

Se realizaron 49 procedimientos, se excluyeron cuatro (tres enteroscopias intraoperatorias y una realizada en un menor de 18 años); por tanto, se incluyeron 45 enteroscopias realizadas a 44 pacientes. La mediana de edad fue 58 años (RIC: 48,5-70,5 años) con un mínimo de 18 años y un máximo de 83 años. La mayoría eran mujeres $(n=29,65,9 \%)$.

La principal indicación fue la hemorragia digestiva potencial del intestino delgado $(n=24,53,3 \%)$ seguida de diarrea crónica $(\mathrm{n}=5,11,1 \%)$ y dolor abdominal crónico $(\mathrm{n}=4,8,9 \%)$ (Tabla 1). El tiempo promedio del procedimiento fue de 90,2 minutos (DE: 23,6 minutos), con un mínimo de 30 minutos y un máximo de 150 minutos.

Tabla 1. Indicaciones y tiempo del procedimiento de EDB

\begin{tabular}{ll}
\hline \multicolumn{1}{|c}{ Variable } & $\mathbf{n}(\%)$ \\
\hline Indicación & \\
\hline $\begin{array}{l}\text { - Hemorragia digestiva potencial del intestino } \\
\text { delgado }\end{array}$ & \\
\hline - Diarrea crónica & $5(53,3)$ \\
- Dolor abdominal crónico & $5(11,1)$ \\
\hline - Enfermedad de Crohn & $4(8,9)$ \\
\hline - Engrosamiento del íleon & $3(6,7)$ \\
\hline - Poliposis intestinal (PAF y síndrome de Peutz- & $2(4,4)$ \\
\hline Jeghers) & $2(4,4)$ \\
\hline - Estenosis (yeyuno y válvula ileocecal) & $2(4,4)$ \\
\hline - Tumor en el intestino delgado & $2(4,4)$ \\
\hline - Yeyunitis en estudio & $1(2,2)$ \\
\hline Tiempo del procedimiento en minutos-media (DE) & $90,2(23,6)$ \\
\hline
\end{tabular}

PAF: poliposis adenomatosa familiar.

Se realizaron 28 procedimientos por vía anterógrada y 17 por vía retrógrada. La longitud mediana alcanzada por vía anterógrada fue $310 \mathrm{~cm}$ (RIC: $222-400 \mathrm{~cm}$ ) y por vía retrógrada fue $195 \mathrm{~cm}$ (RIC: $176-475 \mathrm{~cm}$ ).

La mayoría de los procedimientos fue normal $(\mathrm{n}=21$, $46,7 \%)$; entre los hallazgos más frecuentes estuvieron los tumores $(n=5,11,1 \%)$, enfermedad de Crohn $(n=4,8,9 \%)$ y úlceras/erosiones ( $\mathrm{n}=3,6,7 \%)$ (Tabla 2). En cinco casos $(11,1 \%)$ la enteroscopia diagnosticó lesiones que se encontraban al alcance de estudios endoscópicos convencionales, ya sea por sangrado intermitente o lesiones no diagnosticadas. Un paciente presentaba hernia hiatal gigante con úlceras de Cameron que fue llevado a cirugía 11 meses después del diagnóstico. Dos pacientes presentaron hemorragia secundaria a divertículo de colon que se manejaron endoscópicamente con seguimiento a 14 y 71 meses sin nuevos episodios de hemorragia y uno presentó angiectasia de ciego, que se manejó con electrocoagulación y con seguimiento a 56 meses sin nuevos episodios de hemorragia. El quinto caso fue un paciente inmunosuprimido por síndrome linfoproliferativo que presentó úlcera inflamatoria por histología en el recto; la anemia se corrigió al controlar la patología de base con seguimiento a 69 meses.

Tabla 2. Diagnóstico según EDB

\section{Diagnóstico}

n (\%)

Normal

$21(46,7)$

Tumor

$5(11,1)$

Enfermedad de Crohn

$4(8,9)$

Úlcera/erosión

$3(6,7)$

Divertículo ulcerado

$2(4,4)$

Pólipos

$2(4,4)$

Poliposis intestinal

$2(4,4)$

Angiectasia en el ciego

$1(2,2)$

Enteritis isquémica

$1(2,2)$

Estenosis del yeyuno secundario a bridas

$1(2,2)$

Granuloma inflamatorio posquirúrgico en el yeyuno medio

Hernia hiatal gigante con presencia de úlceras de

Cameron

Lipoma en la segunda porción duodenal

El rendimiento diagnóstico global de la EDB fue del $53,3 \%(\mathrm{n}=24$ hallazgos anormales). Para la entidad más frecuente, hemorragia digestiva potencial del intestino delgado, este rendimiento fue del $54,2 \%$. La correlación de los resultados con la VCE fue del 37,5\% y con estudios de imagen fue del 42,9\%; considerando cualquiera de los dos fue del $40 \%$ (Tabla 3). No se presentaron complicaciones. 
Tabla 3. Correlación de EDB con otros estudios

\begin{tabular}{|lccc|}
\hline \multicolumn{1}{|c}{ Estudio } & $\begin{array}{c}\text { VCE } \\
(\mathbf{n}=16)\end{array}$ & $\begin{array}{c}\text { Estudios } \\
\text { imagenológicos* } \\
(\mathbf{n}=14)\end{array}$ & $\begin{array}{c}\text { Todos los } \\
\text { estudios } \\
(\mathbf{n}=\mathbf{3 0})\end{array}$ \\
\hline $\begin{array}{l}\text { Enteroscopia de } \\
\text { doble balón }\end{array}$ & $6(37,5 \%)$ & $6(42,9 \%)$ & $12(40 \%)$ \\
\hline
\end{tabular}

*TAC, enteroTAC, enterorresonancia, tránsito intestinal. TAC: tomografía axial computarizada.

El seguimiento se consiguió en el 91,1\% ( $\mathrm{n}=41)$; en un tiempo mediano de 56 meses, con un mínimo de 4 y un máximo de 87 meses. La mayoría de estos pacientes se encontraba asintomática y no requirió estudios adicionales $(\mathrm{n}=32,78,1 \%)$; cuatro de los pacientes fallecieron por causas no relacionadas con el procedimiento (Tabla 4). De los 24 pacientes con hemorragia digestiva potencial del intestino delgado se documentaron hallazgos anormales en 13 , de los cuales $2(15,4 \%)$ tuvieron un nuevo episodio de sangrado, mientras que de los 11 con resultados normales solo 1 (9\%) presentó un nuevo episodio de sangrado que en la valoración por coloproctología se consideró secundario a fisura anal.

Tabla 4. Seguimiento de pacientes llevados a enteroscopia de doble balón

\begin{tabular}{|lc|}
\hline \multicolumn{1}{|c|}{ Seguimiento } & n (\%) \\
\hline Pacientes en seguimiento & $41(91,1)$ \\
\hline Tiempo de seguimiento en meses & $\begin{array}{c}\text { Mediana: } 56 \\
\text { RIC: } 15-65 \\
\text { Mínimo: } 4 \\
\text { Máximo: } 87\end{array}$ \\
\hline \multicolumn{1}{|c|}{ Hallazgos } & \\
\hline Resolución del cuadro clínico & $32(78,1)$ \\
\hline Persistencia del cuadro clínico & $5(12,2)$ \\
\hline Fallecimiento & $4(9,8)$
\end{tabular}

A quienes se les realizó patología $(\mathrm{n}=21)$, el resultado principal fue cáncer ( 5 pacientes) e inflamación crónica inespecífica ( 5 pacientes), seguido por enteritis infecciosa $(3$ pacientes) (Tabla 5). Solo el 15,6 \% ( $\mathrm{n}=7)$ de las EDB fue terapéutico; el 84,4\% ( $\mathrm{n}=38)$ tenía indicación diagnóstica.

\section{DISCUSIÓN}

Este estudio describe las indicaciones, hallazgos, complicaciones y relación con otros métodos diagnósticos de la EDB. También describe el seguimiento a los pacientes y busca comparar los resultados con los reportados nacio-
Tabla 5. Resultados de la patología

\begin{tabular}{lc}
\hline \multicolumn{1}{c}{ Resultados } & $\mathbf{n = 2 1}(\mathbf{\%})$ \\
\hline Cáncer & $5(23,8)$ \\
\hline Inflamación crónica inespecífica & $5(23,8)$ \\
\hline Enteritis infecciosa & $3(14,3)$ \\
\hline Pólipos hiperplásicos & $2(9,5)$ \\
\hline Adenoma velloso con displasia de bajo grado & $1(4,8)$ \\
\hline Enfermedad de Crohn & $1(4,8)$ \\
\hline Hiperplasia de las glándulas de Brunner & $1(4,8)$ \\
\hline Pólipos hamartomatosos & $1(4,8)$ \\
\hline Enfermedad celíaca & $1(4,8)$ \\
\hline Normal & $1(4,8)$
\end{tabular}

nal e internacionalmente. A nivel local se han publicado, hasta la fecha, una serie de casos de pacientes llevados a enteroscopia de un solo balón ${ }^{(1)}$ y otra de $\mathrm{EDB}^{(19)}$; asimismo, recientemente se publicó la experiencia específica en hemorragia ${ }^{(20)}$. La mediana de edad en este estudio fue similar a las series reportadas de $\mathrm{EDB}^{(5,21-24)}$.

La hemorragia digestiva potencial del intestino delgado fue también la indicación más frecuente de EDB en otras series de casos, incluidos los publicados en Latinoamérica ${ }^{(3,5,19,21,22,24,25)}$ y en una revisión que incluyó 66 estudios con 12823 procedimientos publicados entre 2001 y $2010^{(26)}$. Lo anterior permite definir que es un método para el diagnóstico y tratamiento de esta entidad.

La vía de inserción varía según la localización más probable de la lesión, determinada principalmente por la historia clínica asociada con estudios de imagen como la VCE. La vía anterógrada fue la más frecuente, y esta es la más empleada también en las series de casos reportadas ${ }^{(5,21,23,24)}$. Lo anterior obedece a que, si se desconoce la ubicación de una lesión, generalmente se utiliza la vía anterógrada porque la técnica retrógrada es más compleja, requiere de preparación, se logra explorar menos intestino y en ocasiones no se logra canalizar la válvula ileocecal ${ }^{(22)}$.

La distancia mediana alcanzada por vía anterógrada fue similar a la reportada mundialmente ${ }^{(13,23)}$. La distancia mediana alcanzada por vía retrógrada aparentemente fue mayor a lo reportado en la literatura ${ }^{(10,24)}$; es importante anotar que estas medidas en todas las series son subjetivas, no homogéneas $y$, por tanto, inexactas, por lo que son difíciles las comparaciones. La tasa y el impacto clínico de la visualización completa del intestino delgado son contro- 
$\operatorname{vertidos}^{(27-29)}$, parece existir consenso en que esto no garantiza un mayor rendimiento diagnóstico o terapéutico ${ }^{(30)}$.

El tiempo promedio del procedimiento fue de 90,2 minutos, y estos son muy variables (40-180 minutos) ${ }^{(3,24,31)}$, debido a distintos factores tales como el entrenamiento y experiencia del gastroenterólogo, antecedente de cirugía previa, adherencias intestinales y obesidad ${ }^{(30)}$.

A pesar de la frecuencia de hallazgos normales ${ }^{(26)}$, no se debe descartar el beneficio de la EDB, dado que la posibilidad de tratamiento que esta ofrece reduce la necesidad de intervenciones más invasivas y riesgos quirúrgicos; además, es un método seguro con bajos índices de complicaciones ${ }^{(22)}$. La tasa de detección de hallazgos anormales puede incrementarse con una rigurosa selección de pacientes y con la realización del procedimiento de manera precoz ${ }^{(32)}$.

La VCE tiene un rendimiento diagnóstico variable (entre el $45 \%$ y el $81 \%$ ), con una tasa de precisión hasta del $30 \%$; se acepta que la EDB es un complemento a las limitaciones de la misma ${ }^{(3)}$. En este estudio, el rendimiento diagnóstico global, definido como el porcentaje de hallazgos anormales, fue del 53,3\%, menor al obtenido en otros estudios ${ }^{(3,21)}$. Este resultado puede explicarse en la realización tardía de la EDB debido a asuntos administrativos relacionados con nuestro sistema de salud o sobrediagnósticos de los estudios previos que incrementan la cantidad de EDB que no se tendrían que realizar.

El rendimiento diagnóstico para hemorragia digestiva potencial del intestino delgado fue $54,2 \%$, similar a otros estudios $^{(4)}$; sin embargo, fue bajo al compararlo con series con resultados cercanos al $80 \%{ }^{(3,31)}$. Esta diferencia puede explicarse debido al tiempo prolongado desde el inicio de los síntomas hasta la realización de los estudios complementarios por razones administrativas ya anotadas ${ }^{(4,16,33,34)}$.

Los diagnósticos más frecuentes en pacientes con hemorragia digestiva potencial del intestino delgado fueron los tumores (12,5\%: dos tumores del estroma gastrointestinal (GIST) y un adenocarcinoma de intestino delgado), seguidos por divertículos de colon ulcerados (8,3\%); estos resultados son similares a lo reportado en la literatura mundial ${ }^{(4)}$.

Entre las fortalezas del estudio está el seguimiento realizado al $91 \%$ de los pacientes en un tiempo prolongado (mediana de 56 meses). El $78 \%$ de los pacientes se encontraba asintomático y no requirió estudios adicionales. Entre las explicaciones a este hallazgo puede sugerirse que las patologías eran alteraciones intermitentes, por lo cual la enteroscopia pudo no ser necesaria o posiblemente los pacientes hayan recibido algún tipo de intervención médica como suspensión de ácido acetilsalicílico (ASA), antiinflamatorios no esteroideos (AINE), antibióticos, anticoagulantes, entre otros, lo que llevó a una resolución de los síntomas. El seguimiento indagó por la resolución de síntomas y realización de estudios adicionales; el estudio se planteó como una serie de casos con un tamaño de muestra reducido, por lo que se requieren estudios de cohorte para evaluar las hipótesis relacionadas con la resolución de los síntomas. Cuando la EDB tiene un resultado anormal, permite dar un manejo dirigido a la patología; si el resultado es normal, teniendo en cuenta que es el estudio de intestino delgado más avanzado, permite indicar seguimiento.

En relación con los nuevos episodios de sangrado, estos se presentaron en $15,4 \%$ (dos pacientes), uno secundario a divertículo de colon y el otro por anemia secundaria a hernia hiatal gigante. En el $9 \%$ (un paciente) con hallazgos normales que persistió con hematoquecia se documentó como causa fisura anal. Si la EDB reporta hallazgos anormales, existe una posibilidad de hasta el $50 \%$ de resangrado; en caso de reportarse hallazgos normales, la posibilidad de resangrado es solo del $5 \%{ }^{(14)}$. Algunos autores sugieren que hay predictores de sangrado recurrente tales como episodios frecuentes de hemorragia y requerimiento transfusional ${ }^{(14)}$.

Durante el estudio de hemorragia digestiva potencial del intestino delgado, los procedimientos endoscópicos convencionales no fueron útiles en un $14 \%$ de los casos ya sea por sangrado intermitente o lesiones no diagnosticadas (hernia hiatal gigante con presencia de úlceras de Cameron, divertículo ulcerado, angiectasia y úlcera en el recto); en la literatura se reporta hasta un $25 \%$ de lesiones hemorrágicas no diagnosticadas por endoscopia alta o baja convencionales que se puede explicar en preparaciones intestinales inadecuadas o nulas; sin embargo, también es importante insistir en la educación académica de los gastroenterólogos que garantice procedimientos convencionales de calidad evitando la práctica de otros avanzados y costosos innecesariamente ${ }^{(14)}$.

En el presente estudio no se presentaron complicaciones, comparable con otras publicaciones que mencionan una baja frecuencia de pancreatitis y perforación ${ }^{(3,26,35)}$.

La EDB es una herramienta útil en la evaluación del intestino delgado con posibilidad terapéutica, baja tasa de complicaciones y rendimiento diagnóstico que puede llegar hasta el $85 \%$, dependiendo de una rigurosa selección de los pacientes ${ }^{(36,37)}$ y de una disminución de sobrediagnósticos en estudios previos; sugerimos su realización en hospitales universitarios que garantizan un entrenamiento y curva de aprendizaje objetiva ${ }^{(15,38,39)}$. El rendimiento diagnóstico para sangrado depende del intervalo de tiempo entre la indicación del procedimiento y su realización; esto solo se logra a partir de un adecuado entendimiento por parte del paciente de su problema, conocimiento académico del personal médico y las entidades administrativas que contribuyan a mejorar esta ventana de oportunidad. 
El seguimiento clínico a los pacientes llevados a EDB puede definir la necesidad de un segundo procedimiento, indicación de estudios adicionales o resolución del problema.

\section{Fuente de financiación}

Sin fuente de apoyo financiero.

\section{REFERENCIAS}

1. Ospina Nieto J, Villamizar Suárez J. Enteroscopia de un balón: Una nueva herramienta para la evaluación gastrointestinal. Rev Col Gastroenterol. 2009;24(3):241-7.

2. Reyes Medina GA. El intestino delgado: ya no es más la "caja negra" del tubo digestivo. Rev Col Gastroenterol. 2009;24(3):238-40.

3. Tang L, Huang LY, Cui J, Wu CR. Effect of Double-Balloon Enteroscopy on Diagnosis and Treatment of Small-Bowel Diseases. Chin Med J (Engl). 2018;131(11):1321-1326. https://doi.org/10.4103/0366-6999.232802

4. Westerhof J, Weersma RK, Koornstra JJ. Investigating obscure gastrointestinal bleeding: capsule endoscopy or double balloon enteroscopy? Neth J Med. 2009;67(7):260-5.

5. De Simone F, Machado P, Guzmán Calderon E. Abordaje diagnóstico y terapéutico de las patologías del intestino delgado mediante cápsula endoscópica y enteroscopía de doble balón. Rev Gastroenterol Perú. 2017;37(1):58-64.

6. Otani K, Watanabe T, Shimada S, Hosomi S, Nagami Y, Tanaka F, Kamata N, Taira K, Yamagami H, Tanigawa T, Shiba M, Fujiwara Y. Clinical Utility of Capsule Endoscopy and Double-Balloon Enteroscopy in the Management of Obscure Gastrointestinal Bleeding. Digestion. 2018;97(1):52-58. https://doi.org/10.1159/000484218

7. Muniraj T, Lee LS. Enteroscopy for GI Fellows. En: Adler D (editor). Upper Endoscopy for GI Fellows. Springer; 2017. p. 151-62. https://doi.org/10.1007/978-3-319-49041-0_13

8. Leighton JA. The role of endoscopic imaging of the small bowel in clinical practice. Am J Gastroenterol. 2011;106(1):27-36; quiz 37. https://doi.org/10.1038/ajg.2010.410

9. Baniya R, Upadhaya S, Subedi SC, Khan J, Sharma P, Mohammed TS, Bachuwa G, Jamil LH. Balloon enteroscopy versus spiral enteroscopy for small-bowel disorders: a systematic review and meta-analysis. Gastrointest Endosc. 2017;86(6):997-1005. https://doi.org/10.1016/j.gie.2017.06.015

10. Rahmi G, Samaha E, Vahedi K, Ponchon T, Fumex F, Filoche B, Gay G, Delvaux M, Lorenceau-Savale C, Malamut G, Canard JM, Chatellier G, Cellier C. Multicenter comparison of double-balloon enteroscopy and spiral enteroscopy. J Gastroenterol Hepatol. 2013;28(6):992-8. https://doi.org/10.1111/jgh.12188

11. Kim TJ, Kim ER, Chang DK, Kim YH, Hong SN. Comparison of the Efficacy and Safety of Single- versus Double-Balloon Enteroscopy Performed by Endoscopist Experts in Single-
Balloon Enteroscopy: A Single-Center Experience and MetaAnalysis. Gut Liver. 2017;11(4):520-527. https://doi.org/10.5009/gnl16330

12. Wadhwa V, Sethi S, Tewani S, Garg SK, Pleskow DK, Chuttani R, Berzin TM, Sethi N, Sawhney MS. A meta-analysis on efficacy and safety: single-balloon vs. double-balloon enteroscopy. Gastroenterol Rep (Oxf). 2015;3(2):148-55. https://doi.org/10.1093/gastro/gov003

13. Lipka S, Rabbanifard R, Kumar A, Brady P. Single versus double balloon enteroscopy for small bowel diagnostics: a systematic review and meta-analysis. J Clin Gastroenterol. 2015;49(3):177-84. https://doi.org/10.1097/MCG.0000000000000274

14. Raju GS, Gerson L, Das A, Lewis B; American Gastroenterological Association. American Gastroenterological Association (AGA) Institute technical review on obscure gastrointestinal bleeding. Gastroenterology. 2007;133(5):1697-717. https://doi.org/10.1053/j.gastro.2007.06.007

15. ASGE Technology Committee, Chauhan SS, Manfredi MA, Abu Dayyeh BK, Enestvedt BK, Fujii-Lau LL, Komanduri S, Konda V, Maple JT, Murad FM, Pannala R, Thosani NC, Banerjee S. Enteroscopy. Gastrointest Endosc. 2015;82(6):975-90. https://doi.org/10.1016/j.gie.2015.06.012

16. Gerson LB, Fidler JL, Cave DR, Leighton JA. ACG Clinical Guideline: Diagnosis and Management of Small Bowel Bleeding. Am J Gastroenterol. 2015;110(9):126587; quiz 1288. https://doi.org/10.1038/ajg.2015.246

17. Pennazio M, Spada C, Eliakim R, Keuchel M, May A, Mulder CJ, Rondonotti E, Adler SN, Albert J, Baltes P, Barbaro F, Cellier C, Charton JP, Delvaux M, Despott EJ, Domagk D, Klein A, McAlindon M, Rosa B, Rowse G, Sanders DS, Saurin JC, Sidhu R, Dumonceau JM, Hassan C, Gralnek IM. Small-bowel capsule endoscopy and deviceassisted enteroscopy for diagnosis and treatment of smallbowel disorders: European Society of Gastrointestinal Endoscopy (ESGE) Clinical Guideline. Endoscopy. 2015;47(4):352-76. https://doi.org/10.1055/s-0034-1391855

18. Enns RA, Hookey L, Armstrong D, Bernstein CN, Heitman SJ, Teshima C, Leontiadis GI, Tse F, Sadowski D. Clinical Practice Guidelines for the Use of Video Capsule Endoscopy. Gastroenterology. 2017;152(3):497-514. https://doi.org/10.1053/j.gastro.2016.12.032 
19. Rey A, Reyes G, Sierra F, Patarroyo O, Ardila A, Prada L. Enteroscopia de doble balón: experiencia de una técnica endoscópica innovadora en el Hospital Universitario de la Fundación Santa Fe de Bogotá. Rev Gastroenterol Mex. 2020;85(1):25-31. https://doi.org/10.1016/j.rgmx.2019.01.001

20. Cañadas Garrido R, Costa Barney VA, Roa Ballestas PA, Espinosa Martínez CA, Pinzón Arenas DF, Ramírez Barranco R. Concordancia diagnóstica entre la videocápsula endoscópica y enteroscopia mono y de doble balón en la hemorragia de intestino delgado en un hospital de alta complejidad en Bogotá, Colombia. Rev Gastroenterol Mex. 2021;86(1):51-8. https://doi.org/10.1016/j.rgmx.2020.03.004

21. Garcia-Correa JJE, Ramirez-Garcia JJ, Garcia-Contreras LF, Fuentes-Orozco C, Irusteta-Jimenez L, Michel-Espinoza LR, Carballo Uribe AS, Torres Chávez JA, GonzálezOjeda A. Double-balloon enteroscopy: Indications, approaches, diagnostic and therapeutic yield, and safety. Early experience at a single center. Rev Gastroenterol Mex. 2018;83(1):31-40. https://doi.org/10.1016/j.rgmxen.2017.08.001

22. Ivano FH, Villela IR, Miranda LF, Nakadomari TS. Analysis of double balloon enteroscopy: Indications, findings, therapeutic and complications. Arq Bras Cir Dig. 2017;30(2):83-7. https://doi.org/10.1590/0102-6720201700020002

23. Dişibeyaz S, Suna N, Kuzu UB, Saygılı F, Öztaş E, Ödemiş B, Önal İK, Kılıç ZM, Akdoğan M, Kayaçetin E. Double balloon enteroscopy: A 7-year experience at a tertiary care Centre. Eur J Intern Med. 2016;33:108-11. https://doi.org/10.1016/j.ejim.2016.06.011

24. Paredes Mendez J, Lazo Molina L, Molina Martos B. Rol de la enteroscopía con doble balón en el manejo de la patología del intestino delgado: experiencia en el Hospital Nacional Guillermo Almenara Irigoyen, Lima, Perú. Rev Gastroenterol Perú. 2016;36(2):107-14.

25. Áñez M, Hernández I, Fuenmayor M, Mengual E, Rangel R, Romero G, Silva N, Namías N, Lizarzábal M. Enteroscopia doble balón y videocápsula endoscópica en el diagnóstico de patologías del intestino delgado: Hospital Universitario de Maracaibo. 2007-2010. Gen. 2011;65(2):117-22.

26. Xin L, Liao Z, Jiang YP, Li ZS. Indications, detectability, positive findings, total enteroscopy, and complications of diagnostic double-balloon endoscopy: a systematic review of data over the first decade of use. Gastrointest Endosc. 2011;74(3):563-70. https://doi.org/10.1016/j.gie.2011.03.1239

27. Lenz P, Domagk D. Double- vs. single-balloon vs. spiral enteroscopy. Best Pract Res Clin Gastroenterol. 2012;26(3):303-13. https://doi.org/10.1016/j.bpg.2012.01.021
28. May A. How much importance do we have to place on complete enteroscopy? Gastrointest Endosc. 2011;73(4):740-2. https://doi.org/10.1016/j.gie.2010.11.030

29. Xin L, Gao Y, Liao Z, Li ZS. The reasonable calculation of complete enteroscopy rate for balloon-assisted enteroscopy. Endoscopy. 2011;43(9):832. https://doi.org/10.1055/s-0030-1256569

30. Jeon SR, Kim JO. Deep enteroscopy: which technique will survive? Clin Endosc. 2013;46(5):480-5. https://doi.org/10.5946/ce.2013.46.5.480

31. Su MY, Lin WP, Chiu CT. Experience of double balloon enteroscopy. J Chin Med Assoc. 2018;81(3):225-9. https://doi.org/10.1016/j.jcma.2017.06.020

32. Tu CH, Kao JY, Tseng PH, Lee YC, Chiang TH, Chen CC, Wang HP, Chiu HM, Wu MS. Early timing of single balloon enteroscopy is associated with increased diagnostic yield in patients with overt small bowel bleeding. J Formos Med Assoc. 2019;118(12):1644-1651. https://doi.org/10.1016/j.jfma.2019.01.003

33. Bresci G, Parisi G, Bertoni M, Tumino E, Capria A. The role of video capsule endoscopy for evaluating obscure gastrointestinal bleeding: usefulness of early use. J Gastroenterol. 2005;40(3):256-9. https://doi.org/10.1007/s00535-004-1532-5

34. Hartmann D, Schmidt H, Bolz G, Schilling D, Kinzel F, Eickhoff A, Huschner W, Möller K, Jakobs R, Reitzig P, Weickert U, Gellert K, Schultz H, Guenther K, Hollerbuhl $\mathrm{H}$, Schoenleben K, Schulz HJ, Riemann JF. A prospective two-center study comparing wireless capsule endoscopy with intraoperative enteroscopy in patients with obscure GI bleeding. Gastrointest Endosc. 2005;61(7):826-32. https://doi.org/10.1016/s0016-5107(05)00372-x

35. Landaeta J, Dias C, Rodríguez M, Urdaneta C. Enteroscopia de doble balón Vs. Enteroscopia de un solo balón en hemorragia digestiva de origen oscuro. Gen. 2010;64(1):26-8.

36. May A. Double-Balloon Enteroscopy. Gastrointest Endosc Clin N Am. 2017;27(1):113-22. https://doi.org/10.1016/j.giec.2016.08.006

37. Moeschler O, Mueller MK. Deep enteroscopy - indications, diagnostic yield and complications. World J Gastroenterol. 2015;21(5):1385-93. https://doi.org/10.3748/wjg.v21.i5.1385

38. Reyes G, Rey M. Entrenamiento en entereoscopia profunda. En: Emura F (editor). ¿Cómo y dónde formarse en los nuevos procedimientos endoscópicos digestivos avanzados, ahora y en el futuro? Cartagena: SIED; 2016. p. 83-5.

39. Gross SA, Stark ME. Initial experience with doubleballoon enteroscopy at a U.S. center. Gastrointest Endosc. 2008;67(6):890-7. https://doi.org/10.1016/j.gie.2007.07.047 